\title{
Causes of Death Data in the Global Burden of Disease Estimates for Ischemic and Hemorrhagic Stroke
}

\author{
Thomas Truelsen $^{\mathrm{a}}$ Lars-Henrik Krarup ${ }^{\mathrm{a}}$ Helle K. Iversen ${ }^{\mathrm{a}}$ George A. Mensah ${ }^{\mathrm{b}}$ \\ Valery L. Feigin ${ }^{\mathrm{e}}$ Luciano A. Sposato $^{\mathrm{d}}$ Mohsen Naghavi $^{\mathrm{C}}$ \\ ${ }^{a}$ Department of Neurology, University of Copenhagen Rigshospitalet, Copenhagen, Denmark; ${ }^{b}$ Center for Translation \\ Research and Implementation Science and Division of Cardiovascular Sciences; National Heart, Lung, and Blood \\ Institute; National Institutes of Health, Bethesda, Md., ' Institute for Health Metrics and Evaluation University of \\ Washington, Seattle, Wash., USA; ${ }^{d}$ Department of Clinical Neurological Sciences, London Health Sciences Centre,

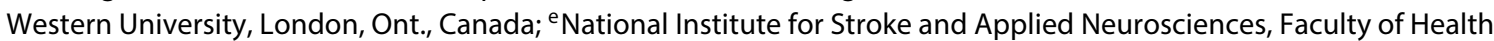 \\ and Environmental Sciences, Auckland University of Technology, Auckland, New Zealand
}

\section{Key Words}

Stroke $\cdot$ Cerebrovascular diseases · Epidemiology $\cdot$ Ischemic stroke $\cdot$ Hemorrhagic stroke

\begin{abstract}
Background: Stroke mortality estimates in the Global Burden of Disease (GBD) study are based on routine mortality statistics and redistribution of ill-defined codes that cannot be a cause of death, the so-called 'garbage codes' (GCs). This study describes the contribution of these codes to stroke mortality estimates. Methods: All available mortality data were compiled and non-specific cause codes were redistributed based on literature review and statistical methods. IIIdefined codes were redistributed to their specific cause of disease by age, sex, country and year. The reassignment was done based on the International Classification of Diseases and the pathology behind each code by checking multiple causes of death and literature review. Results: Unspecified stroke and primary and secondary hypertension are leading contributing 'GCs' to stroke mortality estimates for hemor-
\end{abstract}

rhagic stroke (HS) and ischemic stroke (IS). There were marked differences in the fraction of death assigned to IS and HS for unspecified stroke and hypertension between GBD regions and between age groups. Conclusions: $A$ large proportion of stroke fatalities are derived from the redistribution of 'unspecified stroke' and 'hypertension' with marked regional differences. Future advancements in stroke certification, data collections and statistical analyses may improve the estimation of the global stroke burden.

๑ 2015 S. Karger AG, Basel

\section{Introduction}

The Global Burden of Diseases (GBD) 2013 results have confirmed that stroke remains a leading cause of mortality and disability-adjusted life years [1]. These results further confirm findings from stroke reviews based on published stroke epidemiological studies that show marked variation in stroke rates between countries with especially high rates in low- and middle-income countries [2,3].

\section{KARGER 125}

(c) 2015 S. Karger AG, Base

$0251-5350 / 15 / 0453-0152 \$ 39.50 / 0$

E-Mail karger@karger.com

www.karger.com/ned 


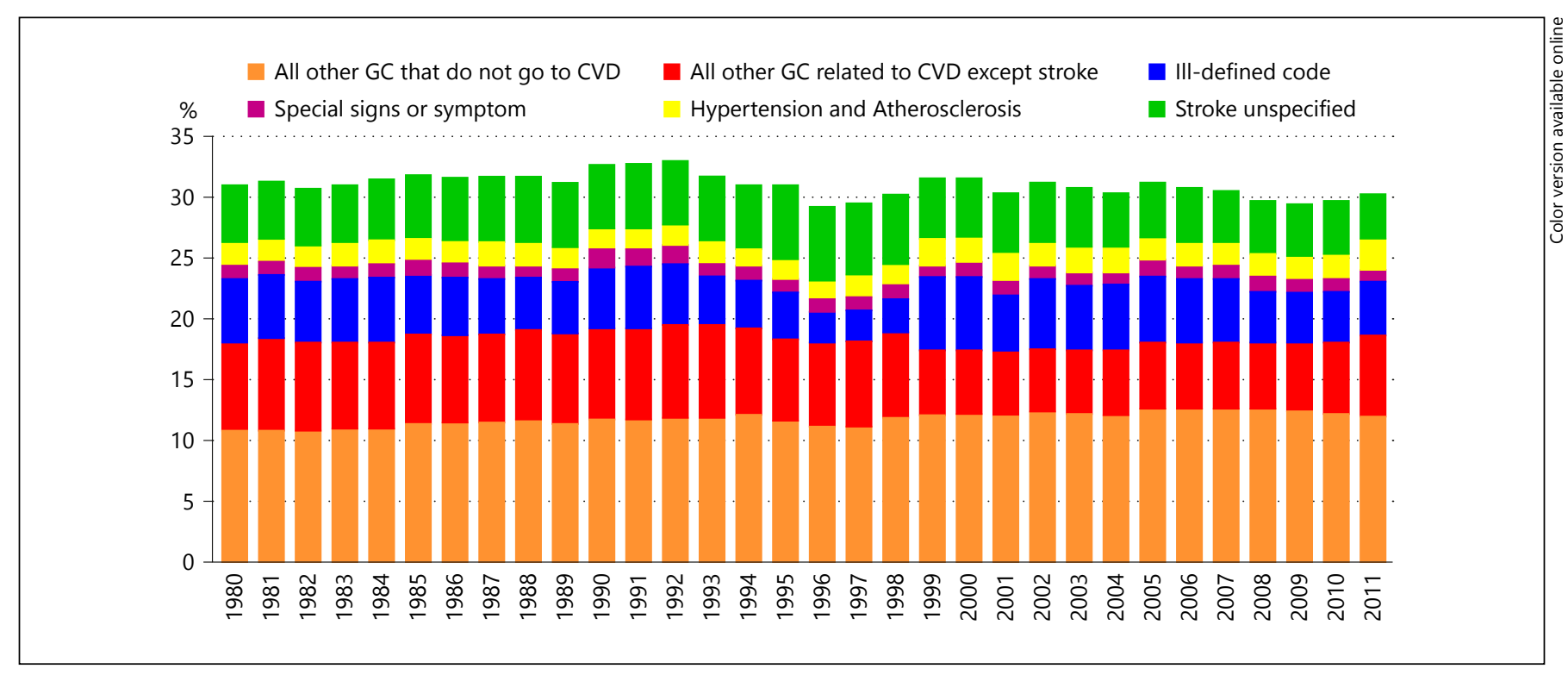

Fig. 1. Fraction of 'GCs' in whole vital registration data with ICD-9 and ICD-10 format at the global level, all ages, men and women combined and by year.

During the nearly 25 years of GBD estimation, there have been significant advancements in the epidemiologic modeling and region/country specific reporting of causes of death (CODs) [4]. The validity of diagnosis of stroke as a COD depends on access to health facilities, local coding practices, availability of CT/MRI imaging, which is of particular importance when dividing the stroke estimates into ischemic stroke (IS) and hemorrhagic stroke (HS) [5, 6]. A key issue in assessing the stroke mortality burden is that varying proportions of unspecified CODs are stroke events that are coded inaccurately, and the handling of these codes may impact estimated stroke mortality and the associated estimations of stroke incidence and prevalence rates. The proportion of routine mortality codes that are unspecific has remained constant at $29-33 \%$ since the launch the GBD, representing the so-called 'garbage codes' (GCs), figure 1 [7].

Every International Classification of Disease (ICD) code that is assigned to death but cannot be a specific underlying cause of death must be redistributed, and during this process, ambiguous codes are split to their likely specific cause. Ill-defined codes are codes that cannot be a cause of death, for example R54 (senility, ICD10). Because these types of GCs are completely ambiguous in the process of CODs estimation, these events are assigned to all specific CODs. Intermediate codes are events coded secondary to a different underlying causes, such as I50 (heart failure, ICD-10). We assigned these events to any cause that pathologically can be underlying cause for this intermediate cause. CODs that can be related to different specific disease categories, for example I10 (hypertension, ICD-10), are assigned to any underlying CODs that pathological and will be directly due to this disorder.

The purpose of the current manuscript is to present the regional distribution of GCs and the impact on regional stroke mortality data for the GBD 2013.

\section{Methodology}

The methodology for the GBD 2013 estimates have been described previously [1]. In brief, all available mortality data were compiled and non-specific cause codes were redistributed based on literature review and statistical methods. The total for all causespecific deaths was fit to an envelope for all-cause mortality for 240 specific causes, including IS and HS.

Specific GCs that have effects on cerebrovascular disease are shown in figure 1 and by age in year 2005 in figure 2. Especially 'ill-defined codes', 'hypertension and atherosclerosis' and 'unspecific stroke' are major contributing codes. ICD codes (ICD-9 and ICD-10) that were used for definition of IS, HS and other GCs are shown in table 1. GCs were redistributed to their specific COD codes (target codes) by age, sex, country and year. Target code reassignment was done one by one based on ICD classification and the pathology behind each GC by checking of multiple CODs and literature review.

We defined the pool of data as all mortality data coded to within the group's undefined codes, or the group's redistribution target 


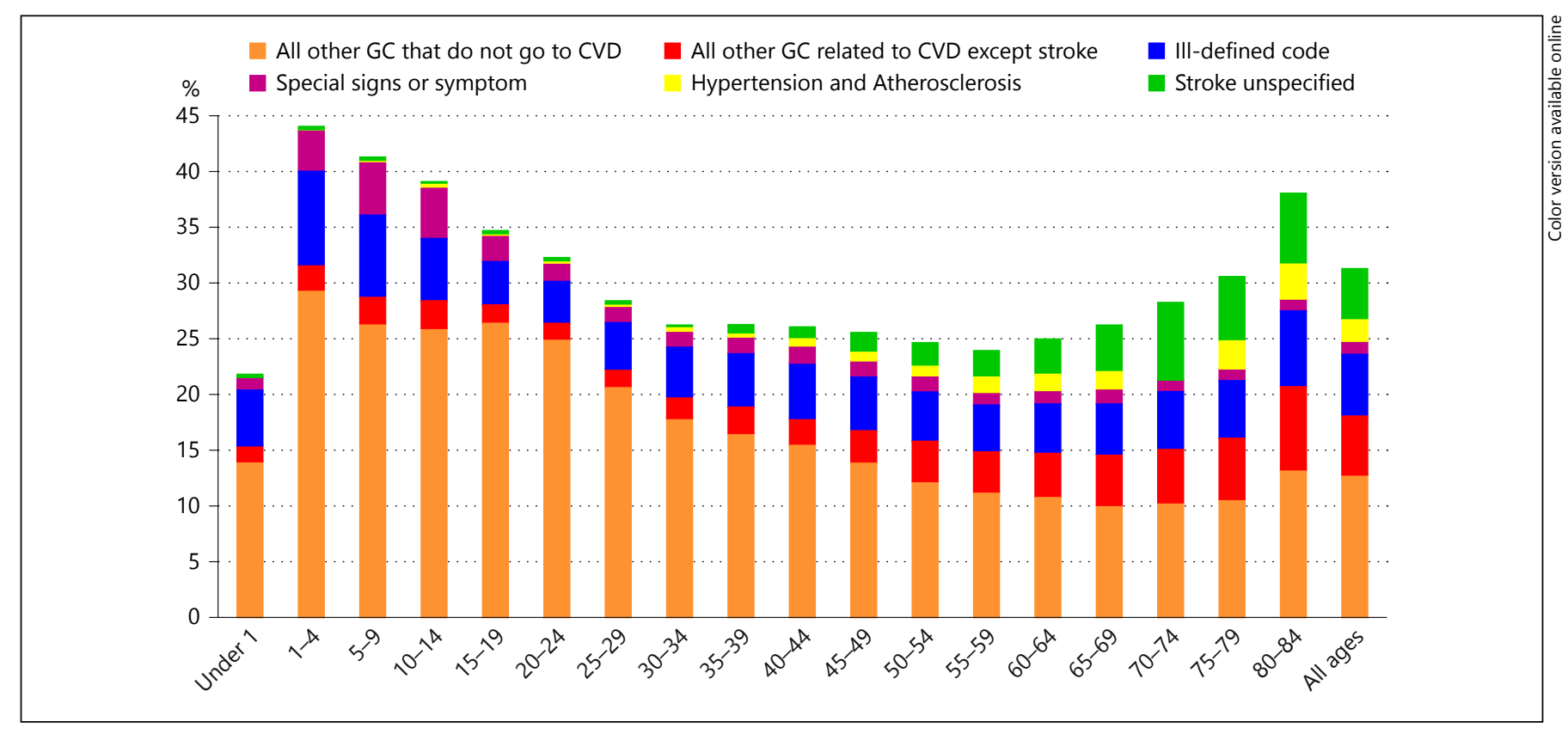

Fig. 2. Fraction of 'GCs' in whole vital registration data with ICD-10 format at the global level, by age, men and women combined, for year 2005.

codes for each country, year, age and sex. We then ran the following regression as described in Ahern et al. [8].

$$
\mathrm{TG}_{\mathrm{crt}}=\alpha=\beta \mathrm{U}_{\mathrm{crt}}+\gamma_{\mathrm{r}}+\theta_{\mathrm{r}} \mathrm{U}_{\mathrm{crt}}+\varepsilon_{\mathrm{ct}}
$$

where $\mathrm{TG}_{\mathrm{crt}}$ : percentage of death data within a given undefined site code's data pool, which were coded to specific site groups, by country $(\mathrm{c})$, year $(\mathrm{t})$ and with countries categorized into regions (r)-: percentage of death data within given undefined site code's coded to undefined site code by country, year, region-fixed constant-: slope coefficient describing associated between and-: region-specific random intercept-: region-specific random slope-: normally-distributed error.

If the coefficient was positive and statistically significant at a $p$ value $<0.05$ level the target group was dropped and the regression rerun. As an example, for the redistribution of the CODs, 'hypertension', 'chronic kidney disease due to hypertension' and 'hypertensive heart disease' were initially included, but for these causes there were not any significant coefficient, and they were consequently omitted.

Furthermore, target codes were dropped if the coefficient was positive and statistically significant. The regression was then repeated a third time. After running the regression 3 times, the $y$ intercept was used as an estimate of an ideal, all-target, no undefined data pool. If there were multiple target groups, the y-intercepts for negative and statistically significant coefficients were scaled to sum $100 \%$, and the proportions for the different target codes were used to redistribute the undefined codes to the target code. We ran these regressions on added whole data set (ICD-9 and ICD-10) for one country (or region) by age and sex. Separate regressions were run for each sex and for the following 3 age groups within each sex: $0-14,15-49$ and $50+$ years.

\section{Results}

The redistribution of GCs to stroke and stroke subtypes varied markedly between regions, age and sex.

For 'unspecified stroke', almost all events in subjects aged $<50$ years are assigned to hemorrhagic stroke and subarachnoid hemorrhage ( $\mathrm{SAH})$; in the CODs estimation, these 2 types of hemorrhages are combined as HS. In subjects aged 70 years or more, mostly all 'unspecified stroke' deaths are assigned to IS, figure 3. Redistribution of 'unspecified stroke' varied by region, as exemplified for men aged 50-69 years, figure 4. In Eastern Europe, almost all unspecified stroke events are assigned to IS whereas the majority of unspecified stroke events in South East and Central Asia are assigned to $\mathrm{ICH}$.

For 'hypertension' as a COD, the redistribution to target codes by age and sex on the global level is shown in figure 5 . In subjects aged less than 15 years, and in women aged 1549 years, HS is the main target code. In men and in subjects aged 50 years or more, the majority of hypertensive deaths are assigned to ischemic heart disease and IS. The regional and global redistribution of deaths coded to hypertension to target codes as exemplified by women aged 50-69 years is shown in figure 6. In Southern Sub-Saharan Africa, more than half of all hypertensive deaths were redistributed to intracranial hemorrhage. The highest proportion of hypertensive deaths assigned to IS occurred in Eastern Europe. 
Fig. 3. Fraction of 'unspecified stroke' assigned to the 3 types of stroke at the global level by age and sex.

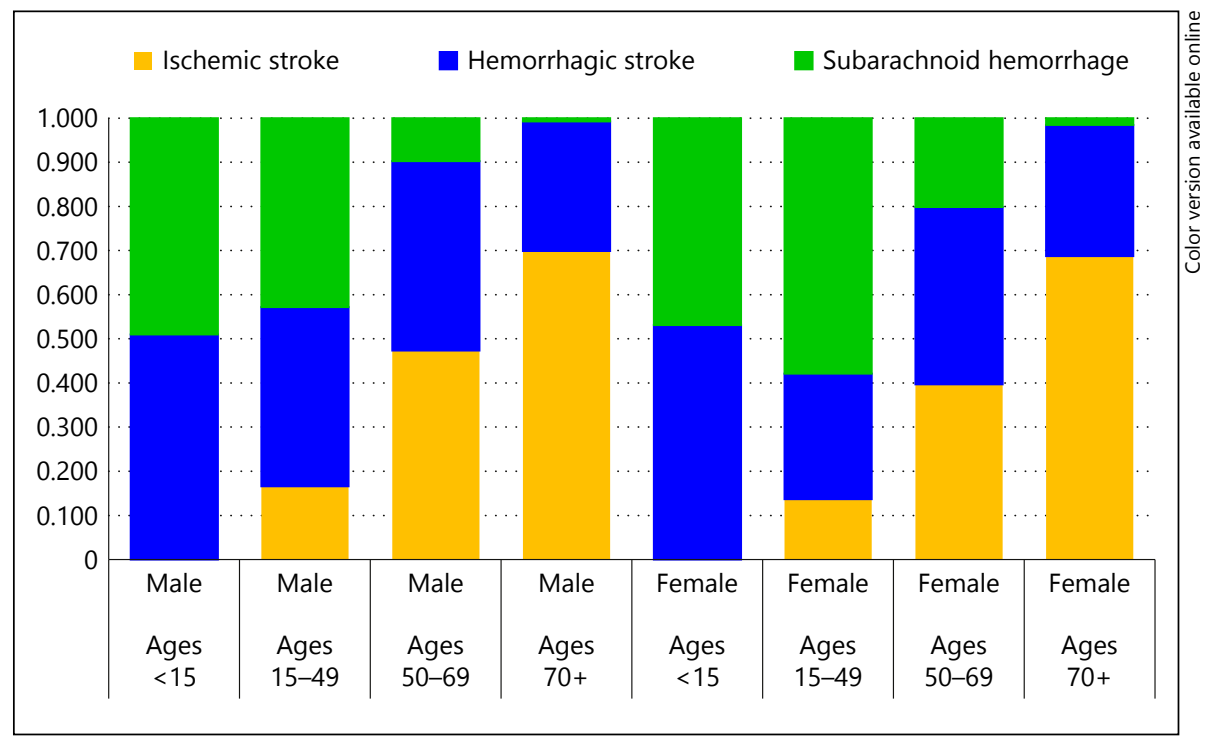

Table 1. Fraction of death coded due to each group of 'GCs' that have been redistributed to each type of stroke, men and women combined, all ages, ICD-10 vital registration

\begin{tabular}{|c|c|c|c|}
\hline Different group of GCs & HS & IS & SAH \\
\hline Stroke unspecified & 30.8 & 64.7 & 4.5 \\
\hline No traumatic extradural hemorrhage & 74.3 & 0.0 & 25.7 \\
\hline Hemiplegia & 29.9 & 54.6 & 9.5 \\
\hline Unspecified disorders of circulatory system in diseases & 46.3 & 18.5 & 22.7 \\
\hline Compression of brain & 33.1 & 0.0 & 31.8 \\
\hline Somnolence, stupor and coma & 31.7 & 27.3 & 5.6 \\
\hline Hypertensive encephalopathy & 17.7 & 27.4 & 13.5 \\
\hline Atherosclerosis & 0.0 & 50.5 & 0.0 \\
\hline Other and unspecified encephalopathy & 0.0 & 0.0 & 38.3 \\
\hline Pneumonitis due to solids and liquids & 23.6 & 14.7 & 0.0 \\
\hline Cerebral edema & 0.0 & 35.9 & 0.0 \\
\hline Primary and secondary hypertension & 6.7 & 29.0 & 0.0 \\
\hline Cardiovascular disease, unspecified & 12.2 & 5.0 & 4.5 \\
\hline Convulsions & 8.4 & 3.0 & 5.8 \\
\hline Age-related physical debility (senility) & 5.8 & 8.6 & 0.7 \\
\hline Unspecified disorder of circulatory system & 6.3 & 3.4 & 0.6 \\
\hline Monoplegia & 4.0 & 4.7 & 1.4 \\
\hline Cardiac arrest, hypotension, syncope and collapse & 4.7 & 4.8 & 0.5 \\
\hline Aphagia and dysphagia & 3.2 & 5.9 & 0.8 \\
\hline Abnormalities of breathing, cyanosis & 4.0 & 4.2 & 1.3 \\
\hline Residual & 3.4 & 3.3 & 0.8 \\
\hline Asphyxia and hypoxemia, respiratory arrest & 3.1 & 3.3 & 0.9 \\
\hline Amnestic disorder and delirium due to known physiological condition & 3.6 & 2.8 & 0.2 \\
\hline Nausea and vomiting & 2.7 & 2.9 & 0.0 \\
\hline All ill-defined codes & 2.2 & 1.7 & 0.6 \\
\hline Dehydration and volume depletion & 1.0 & 1.5 & 0.2 \\
\hline Organ-limited amyloidosis & 0.9 & 0.9 & 0.3 \\
\hline Spastic hemiplegia, paraplegia, tetraplegia & 0.9 & 0.4 & 0.6 \\
\hline Secondary systemic amyloidosis & 0.5 & 0.5 & 0.2 \\
\hline Other disorders of brain and central nervous system & 0.0 & 0.5 & 0.0 \\
\hline Other disorders of brain & 0.0 & 0.5 & 0.0 \\
\hline Pulmonary edema & 0.0 & 0.0 & 0.0 \\
\hline Shock, unspecified & 0.0 & 0.0 & 0.0 \\
\hline Acute respiratory failure, acute respiratory distress syndrome & 0.0 & 0.0 & 0.0 \\
\hline
\end{tabular}

Data are presented as percentages. 
Fig. 4. Fraction of 'unspecified stroke' assigned to the 3 types of stroke by GBD region in men aged $50-69$ years.

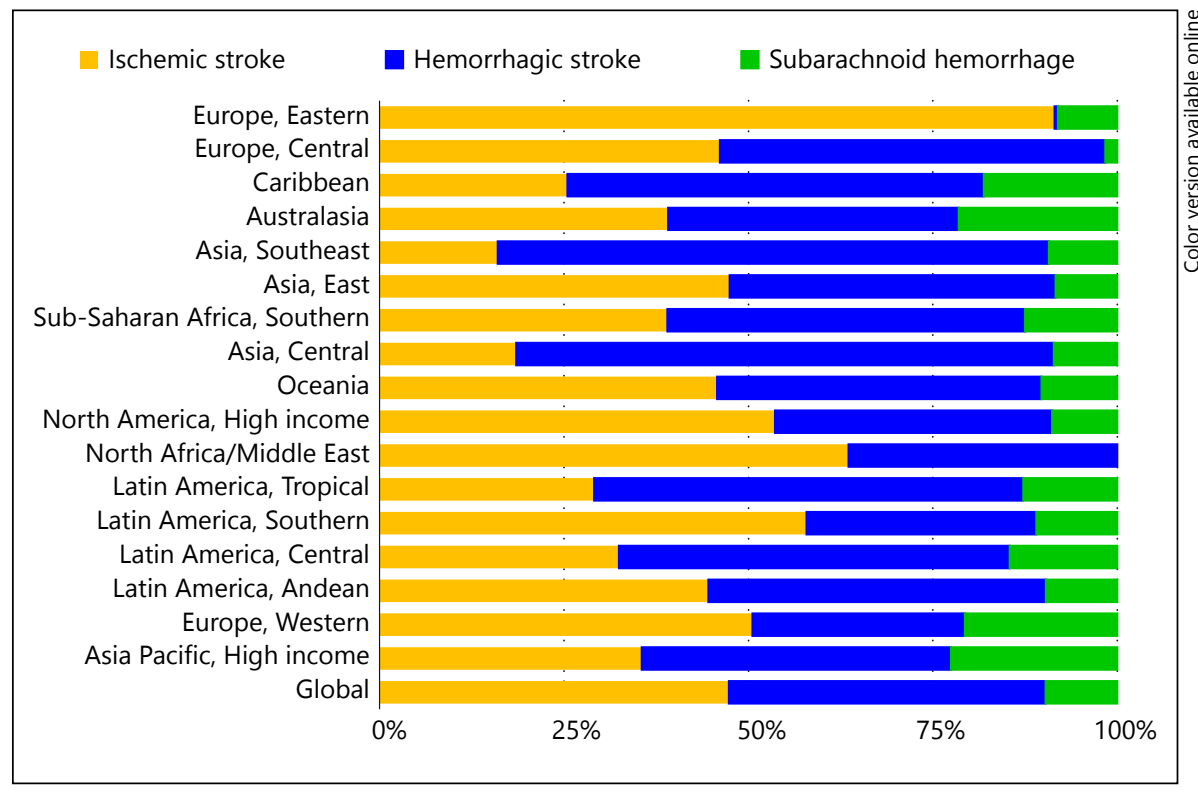

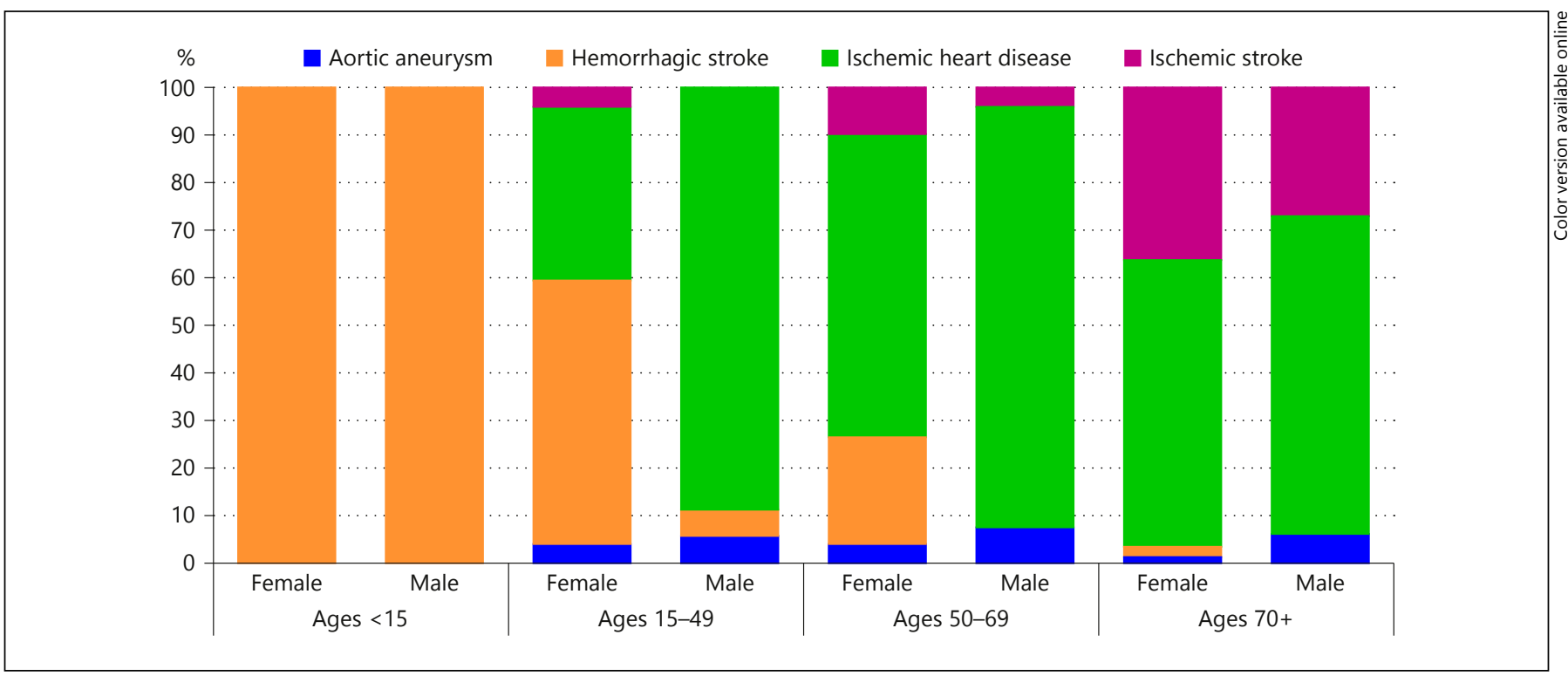

Fig. 5. Fraction of death coded due to hypertension assigned to different target codes by age and sex at the global level.

Other GCs that are redistributed to stroke (ICD-10) are listed in table 1 , and the fractions of deaths that have been added to each of the categories of IS, HS, and SAH are listed in table 2. The complete list of ICD 9 and ICD 10 codes for IS, HS, and associated GCs is provided in table 3. More than half of all IS fatalities and nearly half of all HS fatalities are derived from the redistribution of 'unspecified stroke'.

\section{Discussion}

The GBD provides a unique opportunity for estimating stroke mortality and the associated burden on a global level, applying a standardized methodology taking into account all other major CODs. A large proportion of stroke fatalities stems from the redistribution of especially 'unspecified stroke' and 'hypertension', as well as many other codes. 


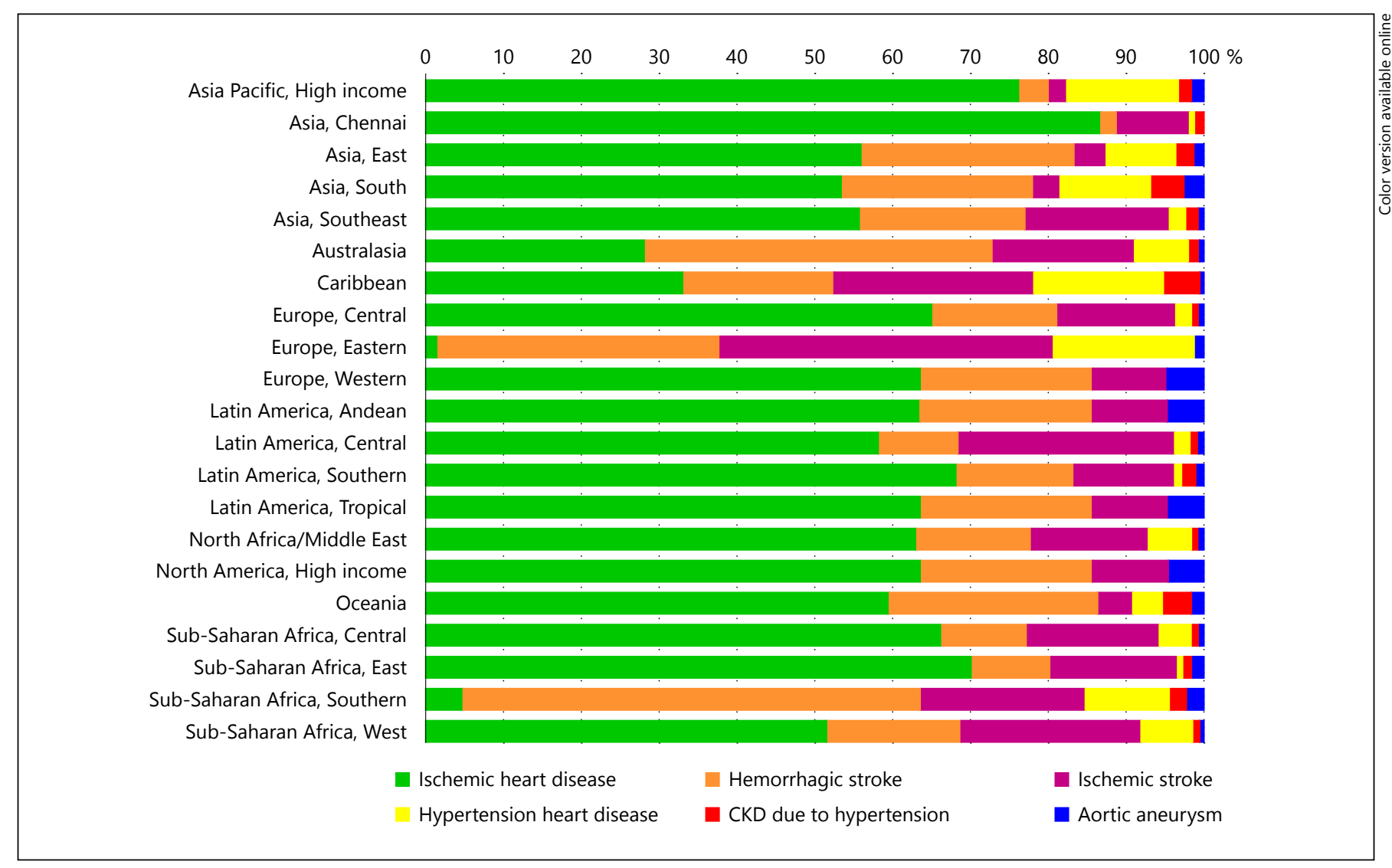

Fig. 6. Fraction of death coded due to hypertension assigned to the different target codes in females aged 50-69 years, regional level.

Table 2. Fraction of death that have been added to each type of stroke after redistribution of 'GCs', men and women combined, all ages, year 2008, ICD-10 vital registration

\begin{tabular}{lrrr}
\hline Different group of GCs & HS & IS & SAH \\
\hline Stroke unspecified & 88.6 & 162.8 & 46.1 \\
Primary and secondary hypertension & 4.7 & 17.7 & 0.0 \\
Atherosclerosis & 0.0 & 21.6 & 0.0 \\
Non-traumatic extradural hemorrhage & 6.0 & 0.0 & 7.4 \\
Age-related physical debility (senility) & 4.9 & 6.3 & 2.0 \\
Pneumonitis due to solids and liquids & 7.1 & 3.8 & 0.0 \\
All ill-defined codes & 2.7 & 1.8 & 2.7 \\
Cardiac arrest, hypotension, syncope and collapse & 2.8 & 2.5 & 1.0 \\
Asphyxia and hypoxemia, respiratory arrest & 0.5 & 0.5 & 0.5 \\
Residual & 0.5 & 0.4 & 0.4 \\
Somnolence, stupor and coma & 0.5 & 0.3 & 0.3 \\
Hypertensive encephalopathy & 0.2 & 0.4 & 0.6 \\
Hemiplegia & 0.3 & 0.5 & 0.3 \\
Cerebral edema & 0.0 & 0.1 & 0.0 \\
Dehydration and volume depletion & 0.1 & 0.1 & 0.1 \\
Unspecified disorder of circulatory system & 0.1 & & \\
\hline
\end{tabular}

Data are presented as percentages. 
Table 3. ICD code for definition of IS, HS and GCs by ICD-9 and ICD-10, 4-digit code level

\begin{tabular}{ll}
\hline $\begin{array}{l}\text { Cause } \\
\text { name }\end{array}$ & ICD-10 code 9 code \\
\hline
\end{tabular}

IS G45-G46.8, I63-I63.9, I65-I66.9, I67.2, I67.3, I67.5, I67.6, I69.3-I69.398

HS I60-I61.9, I62.0-I62.03, I67.0, I67.1, I67.7, I69.0-I69.198, $\mathrm{I} 69.20-\mathrm{I} 69.298$

GC A01, A14.9, A29-A30.9, A40-A41.9, A45, A45.9, A47-A48.0, A48.3, A48.8-A49.02, A49.2-A49.9, A59-A62, A64, A64.0, A71-A73, A74.0, A76, A97, A99, A99.0, B07-B09, B11-B14, B17.8, B17.9, B19, B19.0, B19.9, B28, B29, B30-B32.4, B34B36.9, B55, B55.1-B55.9, B61, B62, B64, B66-B66.9, B73-B74.2, B76-B76.9, B79, B82-B82.9, B83.9-B89, B92-B94.0, B94.8, B94.9, B95.6-B99.9, C14-C14.9, C26-C29, C35, C36, C39C39.9, C42, C46-C46.9, C55-C55.9, C57.9, C59, C63.9, C68, C68.9, C75.9-C80.9, C87, C98-D00.0, D01, D01.4-D02, D02.4, D02.9, D07, D07.3-D07.39, D07.6-D09, D09.1-D09.19, D09.9, D10, D10.9, D13, D13.9, D14, D14.4, D17-D21.9, D26, D26.7, D26.9, D28, D28.9, D29, D29.9, D30, D30.9, D36.0, D36.9D37.0, D37.6-D38, D38.6-D39.0, D39.9, D40, D40.9, D41, D41.9, D44, D44.9, D48, D48.7-D49.1, D49.5, D49.7, D49.8, D49.89, D49.9, D54, D59, D59.4, D59.8, D59.9, D64, D65D65.9, D75.9, D79, D84, D84.9, D85, D87, D88, D89.8-D99, E07.8-E08.9, E17-E19, E34.9-E35.8, E37-E39, E47-E50.9, E62, E64.1, E69, E85.3-E87.70, E87.79-E87.99, E90-E99.9, F04F06.1, F06.3-F07.0, F07.2-F09.9, F12-F12.99, F17-F17.9, F24, F30-F50, F50.2-G00, G00.9-G02.8, G03.9, G06-G09.0, G15G19, G27-G29, G32-G34, G38, G39, G42-G44.89, G47-G47.29, G47.4-G60.9, G62-G69, G74-G89.4, G91-G93.6, G93.8-G94.8, G96-G96.9, G98-H69.93, H71-H99, I00.0, I03, I04, I10-I10.9, I14-I19, I26-I27.0, I27.2-I27.9, I28.9-I29.9, I31.2-I31.4, I32I32.8, I43-I46.9, I49-I51, I51.7-I59, I62, I62.1, I62.9, I64-I64.9, I67, I67.4, I67.8-I69, I69.2, I69.4-I70.1, I70.9-I70.92, I79-I79.8, I90, I92-I95.1, I95.8-I96.9, I98.4, I98.8, I99-J00.0, J02, J02.8J03, J03.8-J04.31, J06-J08, J15.9, J17-J19.6, J22-J29, J48-J59, J64-J64.9, J69-J69.9, J71-J81.9, J83, J85-J90.9, J93-J94.9, J96K19, K23-K24, K30, K31.9-K34, K39, K47-K49, K53, K54, K58, K63-K63.4, K63.8-K63.9, K65-K66.1, K66.9, K69, K71-K71.2, K71.6, K71.8-K72.01, K75-K75.1, K78, K79, K84, K87-K89, K92.0-K92.2, K92.9, K93, K93.1, K93.8, K96-K99, L06, L07, L09, L15-L50.9, L52-L87.9, L90-L92.9, L94-L96, L98.5-L99.8, M04, M10-M12.09, M12.2-M29, M37-M39, M43.2-M49, M49.2-M64, M65.1-M71, M71.2-M73, M73.8-M85.9, M87.2M87.9, M89.1-M89.49, M90-M99.9, N09, N13-N13.9, N17N17.9, N19-N19.9, N24, N32.1, N32.2, N32.8-N33.8, N35N35.9, N37-N38, N39.3-N40.9, N42-N43.42, N46-N48.9, N52N64.9, N66-N69, N78, N79, N82-N82.9, N84, N84.2-N86, N88-N95.9, N97-N97.9, O08-O08.9, O17-O19, O27, O37O39, O49-O59, O78, O79, O93-O95.9, P06, P16-P18, P30P34.2, P40-P49, P62-P69, P73, P79, P82, P85-P89, P96.9-P99.9, Q08-Q10.3, Q19, Q29, Q36.0-Q36.9, Q46-Q49, Q88, Q89.9, Q94, Q96-Q96.9, Q98-Q98.9, Q99.9-R19.6, R19.8-R50.1, R50.8, R50.81, R50.84-R72.9, R74-R78, R78.6-R94.8, R95.0T98.3, U04.9, V87-V87.1, V87.4-V88.1, V88.4-V89.9, V90.00, V99, V99.0, W47, W48, W63, W71, W72, W82, W95-W97, W98, X07, X41-X42.9, X44-X44.9, X55, X56, X59-X59.9, Y09Y34.9, Y85-Y87.0, Y87.2, Y89

$433-435.9,437.0,437.1,437.5-437.8$

$430-432.9,437.2$

000, 000.9, 002, 030-030.9, 038-038.9, 039.6, 040.0, 041.9, 054.1, 067-070, 070.4, 070.49, 070.5, 070.59, 070.6, 070.9, 076-076.1, 076.6, 076.9-078.3, 085, 085.1-085.9, 089-089.9, $108-111.9,112.0,112.3-117.2,117.4-117.6,117.8-119$, 121-121.9, 125.0-125.3, 126-126.9, 127.3, 130-134.9, $136.3-136.5,136.8,136.9,139.1,139.8,149-149.9,159-$ 159.9, 165-169, 176-179.9, 183.9, 184, 184.5, 184.9, 187, $187.9,189,189.9,194.9-199.9,209,209.2,209.20,209.29-$ 209.30, 209.6, 209.60, 209.62, 209.69-210, 211, 211.9, 212, $212.9,214-216.9,219,219.8,219.9,221,221.9,222,222.9$, 223, 223.9, 229, 229.1, 229.9-230.0, 230.9, 231, 231.8, 231.9, $233,233.3,233.30,233.39,233.6,233.9,234,234.9,235$, 235.1-235.3, 235.5, 235.9-236.0, 236.3, 236.6, 236.9, 236.90, $238,238.9-239.1,239.5,239.7-239.9,244,244.9,247-$ 249.91, 264-264.9, 274-274.9, 276.0-276.9, 277.3, 278, 279.0-279.53, 279.8, 279.9, 286.6, 293-294.0, 296-302.9, $304,304.3-304.33,304.9-304.93,305.2-305.23,306-307.0$, $307.2-307.53$, 307.59-320, 320.9, 321.0, 324-327.19, 328, $329,331.3,331.4,338-339.89,342-344.9,346-348.9$, $349.81-353.5,354-355.9,357,357.8-357.9,360-380.9$, 384-389.9, 399-401.9, 405-409.4, 415-416.0, 416.3-416.9, $418-419.9,423.0,426-426.9,427.4-427.5,427.9-429$, 429.2-429.9, 436-437, 437.3, 437.4, 437.9-440.9, 458-458.9, 459.0, 459.5-460.9, 462-465.9, 482.9, 483, 484, 484.8-486.9, 505-505.9, 507-507.9, 510-514.9, 515.0, 515.9, 518-518.53, 518.8-518.89, 519, 519.9-529.9, 536.2, 536.3, 536.8, 536.9, $537.7,537.89,537.9,544-549,553.8,553.9,559,559.0$, $560.4-560.7,561,562.2-563,564.8-564.9,567-569,569.49$, $569.79-569.83,569.86-570.9,572-572.2,573,573.5,578-$ $578.9,584-584.9,586-587.9,591-591.9,593.9,599.60$ $599.72,599.9-600.91,603-603.9,605-609,611-612.1,615-$ $616.9,618-619.9,621-621.35,622-622.2,622.8-628.9$, $629.89,629.9,637-637.92,639-639.9,690-693.9,695.8-$ 706.9, 708-709.9, 712-713.8, 714.4, 715-716, 716.1-728.85, $728.87,728.89-729.99,730.3-730.39,730.7-730.79,731-$ 731.9, 733-739.9, 749.1-749.14, 758.7, 759, 759.9, 770.0, $779.9-780.56,780.58-780.61,780.64-786.02,786.04-$ 787.04, 787.2-787.9, 787.99, 788, 788.1-790.1, 790.29, 790.4-798, 798.1-999.9, E80, E80.08, E80.09, E80.18, E80.19, E80.28, E80.29, E80.38, E80.39, E80.48, E80.49, E80.58, E80.59, E80.68, E80.69, E80.78-E81.0, E81.08E81.1, E81.18-E81.2, E81.28-E81.3, E81.38-E81.4, E81.48E81.5, E81.58-E81.6, E81.68-E81.7, E81.78-E81.8, E81.88E81.9, E81.98-E82.0, E82.08-E82.1, E82.18-E82.2, E82.28E82.3, E82.38-E82.4, E82.48-E82.5, E82.58-E82.6, E82.68E82.7, E82.78-E82.8, E82.88-E82.9, E82.98-E83, E83.9, E85, E85.58-E85.599, E85.9, E87, E87.7, E88.7-E88.709, E91.4-E91.409, E92.89-E92.90, E92.95-E92.99, E98.0E98.9, V01-V29.9 
Ideally, the global burden of stroke should be based on the 'gold standard' stroke epidemiology data; however, there is a shortage of such data from high-income countries and, especially, from low- and middle-income countries [3]. In the absence of ideal data, routine mortality statistics are used relying on the certifying person's ability to correctly assess and code what was the underlying cause of death. Previous studies from different countries have shown that the validity of stroke subtype diagnoses varies $[5,6,9]$. Whereas 'IS' and 'intracerebral hemorrhage' codes are often correct, maybe by requiring a detailed level of knowledge of the cause of the subjects' symptoms including access to scanning facilities, there is more uncertainty with regards to the validity of the diagnosis 'unspecified stroke' as this may be based on clinical signs alone without neuroimaging. The access to healthcare and imaging facilities varies between countries as well as between socioeconomic groups between and within countries and could impact death certification and coding practice.

In regions without routine mortality statistics, knowledge about stroke as a COD may be derived from results based on verbal autopsy [10]. There are several examples of such studies from different countries where assessment of stroke has been examined [11-13]. Differences in the development of disease algorithms could hamper the comparability, but represents a first step in increasing the knowledge of cerebrovascular diseases as a COD.

For countries with no data available, like some countries in North Africa, the Middle-East and large parts of Asia, the estimations are based on regional patterns and a full set of covariates that are available for all countries and for all years. Shortage on data causes uncertainty about the redistribution of GCs to target codes. As an example in this study, 'hypertension' was very differently redistributed to ischemic heart disease, IS and HS in Eastern Europe as compared to Central and Western Europe.
Studies have suggested that there are marked differences in the occurrence of different subtypes of stroke between European populations $[14,15]$; the extent to which the current redistribution is consistent for all countries within each region is beyond the scope of this publication. However, it is an example of how increasing knowledge of stroke epidemiology and advancements in the statistical methods for redistributing CGs may lead to improvements in the future GBD methodology. Future results from large-scale population-based studies on stroke incidence, mortality and fatality [16] will be essential for refining regional GBD estimates.

In conclusion, stroke mortality data in the GBD is derived from routine statistics and the redistribution of illdefined and other diagnostic codes, which cannot be considered as specific CODs. A large proportion of stroke fatalities are derived from the redistribution of 'unspecified stroke' and 'hypertension'. Regional differences are marked, which is supported by real-world data; however, future advancements in stroke certification, data collections and statistical analyses may improve the estimation of the stroke mortality burden.

\section{Disclosure Statement}

H.K. Iversen serves on scientific advisory boards for Bayer, Boehringer-Ingelheim, Bristol Myers-Squibb/Pfizer, has received honoraria from Boehringer-Ingelheim, Denmark, Bristol MyersSquibb and Pfizer. Serves on the expert panel for the Danish Stroke Patient Association. The other authors have no conflicts of interest.

\section{Disclaimer}

The views expressed in this article are those of the authors and do not necessarily represent the views of the National Heart, Lung, and Blood Institute; National Institutes of Health or the US Department of Health and Human Services.

\section{References}

1 GBD 2013 Mortality and Causes of Death Collaborators: Global, regional, and national age-sex specific all-cause and cause-specific mortality for 240 causes of death, 1990-2013: a systematic analysis for the global burden of disease study 2013. Lancet 2015;385:117-171.

2 Feigin VL, Forouzanfar MH, Krishnamurthi $\mathrm{R}$, Mensah GA, Connor M, Bennett DA, Moran AE, Sacco RL, Anderson L, Truelsen T, O’Donnell M, Venketasubramanian N, Barker-Collo S, Lawes CM, Wang W, Shino- hara Y, Witt E, Ezzati M, Naghavi M, Murray C; Global Burden of Diseases, Injuries, and Risk Factors Study 2010 (GBD 2010) and the GBD Stroke Experts Group: Global and regional burden of stroke during 19902010: findings from the global burden of disease study 2010. Lancet 2014;383:245254.

3 Feigin VL, Lawes CM, Bennett DA, BarkerCollo SL, Parag V: Worldwide stroke incidence and early case fatality reported in 56 population-based studies: a systematic review. Lancet Neurol 2009;8:355-369.

4 Naghavi M, Makela S, Foreman K, O’Brien J, Pourmalek F, Lozano R: Algorithms for enhancing public health utility of national causes-of-death data. Popul Health Metr 2010; 8:9.

5 Krarup LH, Boysen G, Janjua H, Prescott E, Truelsen T: Validity of stroke diagnoses in a national register of patients. Neuroepidemiology 2007;28:150-154. 
6 Jones SA, Gottesman RF, Shahar E, Wruck L, Rosamond WD: Validity of hospital discharge diagnosis codes for stroke: the atherosclerosis risk in communities study. Stroke 2014;45:3219-3225.

7 Murray JL, Lopez AD (eds): Global Burden of Disease and Injury Series. The Global Burden of Disease: A Comprehensive Assessment of Mortality and Disability from Diseases, Injuries, and Risk Factors in 1990 and Projected to 2020. Boston, Harvard School of Public Health, World Health Organization and the World Bank, 1996, pp 117-200.

8 Ahern RM, Lozano R, Naghavi M, Foreman $\mathrm{K}$, Gakidou E, Murray CJ: Improving the public health utility of global cardiovascular mortality data: the rise of ischemic heart disease. Popul Health Metr 2011;9:8.

9 Malmivaara A, Meretoja A, Peltola $\mathrm{M}, \mathrm{Nu}$ merato D, Heijink R, Engelfriet P, Wild SH, Belicza É, Bereczki D, Medin E, Goude F, Boncoraglio G, Tatlisumak T, Seppälä T, Häkkinen U: Comparing ischaemic stroke in six European countries. The Eurohope regis- ter study. Eur J Neurol 2015;22:284-291.e25e26.

10 Joshi R, Kengne AP, Neal B: Methodological trends in studies based on verbal autopsies before and after published guidelines. Bull World Health Organ 2009;87:678-682.

11 Kalkonde YV, Deshmukh MD, Sahane V, Puthran J, Kakarmath S, Agavane V, Bang A: Stroke is the leading cause of death in rural gadchiroli, India: a prospective community-based study. Stroke 2015;46:17641768.

12 Walker R, Whiting D, Unwin N, Mugusi F, Swai M, Aris E, Jusabani A, Kabadi G, Gray WK, Lewanga M, Alberti G: Stroke incidence in rural and urban tanzania: a prospective, community-based study. Lancet Neurol 2010; 9:786-792.

13 Sepanlou SG, Sharafkhah M, Poustchi $H$, Malekzadeh MM, Etemadi A, Khademi H, Islami F, Pourshams A, Pharoah PD, Abnet CC Brennan P, Boffetta P, Dawsey SM, Esteghamati A, Kamangar F, Malekzadeh R: Hypertension and mortality in the golestan cohort study: a prospective study of 50000 adults in Iran. J Hum Hypertens 2015, Epub ahead of print.

14 Ingall $\mathrm{T}$, Asplund $\mathrm{K}$, Mähönen $\mathrm{M}$, Bonita R WHO MONICA Project: A multinational comparison of subarachnoid hemorrhage epidemiology in the WHO MONICA stroke study. Stroke 2000;31:1054-1061.

15 Truelsen T, Malekzadeh MM, Etemadi A, Khademi $\mathrm{H}$, Islami $\mathrm{F}$, Pourshams A, Pharoah PD, Abnet CC, Brennan P: Boffeca project. Stroke 2003;34:1346-1352.

16 Sposato LA, Coppola ML, Altamirano J, Borrego Guerrero B, Casanova J, De Martino M, Díaz A, Feigin VL, Funaro F, Gradillone ME, Lewin ML, Lopes RD, López DH, Louge M, Maccarone P, Martens C, Miguel M, Rabinstein A, Morasso H, Riccio PM, Saposnik G, Silva D, Suasnabar R, Truelsen T, Uzcudun A, Viviani CA, Bahit MC: Program for the epidemiological evaluation of stroke in Tandil, Argentina (PREVISTA) study: rationale and design. Int J Stroke 2013;8:591597. 